Wright State University

CORE Scholar

$1-1-1996$

\title{
Magnetophotoluminescence Study of Excited States Associated with Donor Bound Excitons in High-Purity GaAs
}

D. C. Reynolds

David C. Look

Wright State University - Main Campus, david.look@wright.edu

B. Jogai

G. L. McCoy

K. K. Bajaj

Follow this and additional works at: https://corescholar.libraries.wright.edu/physics

Part of the Physics Commons

\section{Repository Citation}

Reynolds, D. C., Look, D. C., Jogai, B., McCoy, G. L., \& Bajaj, K. K. (1996). Magnetophotoluminescence

Study of Excited States Associated with Donor Bound Excitons in High-Purity GaAs. Physical Review B, 53 (4), 1891-1895.

https://corescholar.libraries.wright.edu/physics/199

This Article is brought to you for free and open access by the Physics at CORE Scholar. It has been accepted for inclusion in Physics Faculty Publications by an authorized administrator of CORE Scholar. For more information, please contact library-corescholar@wright.edu. 


\title{
Magnetophotoluminescence study of excited states associated with donor bound excitons in high-purity GaAs
}

\author{
D. C. Reynolds, D. C. Look, and B. Jogai \\ University Research Center, Wright State University, Dayton, Ohio 45434 \\ G. L. McCoy \\ Solid State Electronics Directorate, Wright Laboratory, WL/ELR, Wright-Patterson Air Force Base, Ohio 45433 \\ K. K. Bajaj \\ Department of Physics, Emory University, Atlanta, Georgia 30322
}

(Received 29 September 1995)

\begin{abstract}
Detailed magnetic-field splitting of the two rotator states designated as $\left(\Gamma_{6}+\Gamma_{8}, J=\frac{5}{2}\right)$ and $\left(\Gamma_{7}, J=\frac{1}{2}\right)$ associated with donor bound excitons in high-purity epitaxial GaAs is observed in magnetophotoluminescence spectra. These two states are associated with the light-hole mass and are split by the spin-orbit interaction. The ordering in energy of these two states agrees with that predicted by theory. Also observed are the rotator states in which, after the radiative collapse of the exciton, the donor is left in the excited states. A good agreement between the energies of the excited states of the donor as determined experimentally with those calculated using a variational approach is found. The magnetophotoluminescence spectra at relatively low magnetic fields clearly show the evolution of the excited donor bound exciton states as well as the excited rotator states.
\end{abstract}

\section{INTRODUCTION}

Shallow residual donors have been identified in GaAs using photoluminescence (PL) and magnetophotoluminescence (MPL) spectroscopy. ${ }^{1-5}$ The optical transitions that were used to identify the residual donors result from the collapse of neutral donor bound excitons $D^{0}, X$. The exciton bound to the donor may be in the ground state $D^{0}, X$ or in various excited states $\left(D^{0}, X\right)^{*}$ of the $D^{0}, X$ complex. The collapse of the exciton may leave the donor in the ground state $D^{0}$ or in different excited states $D^{0 *}$, other than the ground state $D^{0}$, the transition can terminate in either the $2 S$ or $2 P$ state, considering only the lowest excited states. From parity considerations it can be shown that the initial state of the $D^{0}, X$ has odd parity. The $D^{0 *} 2 S$ final state will have even parity, whereas the $2 P$ final state will have odd parity. The preferential transition will thus be from the $D^{0}, X$ initial state to the $D^{0 *} 2 S$ final state. By similar arguments it can be shown that the initial state of the exciton bound in the first excited state $\left(D^{0}, X\right)^{*}$ will have even parity. The preferential transition from this state will be to the $D^{0 *} 2 P$ final state having odd parity. The intensities of these transitions were studied by Dean, Herbert, and Lahee ${ }^{6}$ for the case of ZnTe. They showed that when the exciton collapsed from the first initial state, the terminal state intensity ratio was $2 P / 2 S=20$. They further showed that, when the exciton collapsed from the initial $D^{0}, X$ ground state, the terminal state intensity ratio was $2 P / 2 S=1 / 5$. From these arguments it can be concluded that the $D^{0 * 2 P}$ final states are predominantly associated with having the initial configuration in the first excited state, and the $D^{0 *} 2 S$ final state is predominantly associated with the $D^{0}, X$ initial ground state. Performing the experiment in the presence of a magnetic field produces two effects: (a) it separates out states with different orbital angular momenta, and (b) it compresses the wave functions, which sharpens the lines and separates the different donor states.

The origin of the initial donor bound exciton excited states has been an intriguing problem for many years, and is still not completely resolved. Guillaume and Lavallard ${ }^{7}$ proposed a rigid rotation model to explain the excited states in the case of CdTe. In this model the hole is excited to rotate around the fixed donor analogous to the rotation of a diatomic molecule. This model had difficulty in predicting the observed energies for the excited-state transitions. A nonrigid rotator model was subsequently proposed by Rühle and Klingenstein ${ }^{8}$ which was successful in predicting the excitedstate energies in InP and GaAs. A more sophisticated model was applied to the $D^{0}, X$ ground and excited states by Herbert. ${ }^{9}$ This model predicts the ordering of the excited states. In Ref. 8 it was shown that the projection of the hole spin on the orbital angular momentum $l=1$ state results in two rotational states, one with a heavy-hole mass and a total angular momentum $J=\frac{3}{2}$. This will be the lowest-energy rotational state. The other state is associated with the light-hole mass, and is split into two energy levels by spin-orbit interaction. One of these states will have a total orbital and angular momentum of $J=\frac{1}{2}$ and the other $J=\frac{5}{2}$. Considering the energy ordering of all of the ground and excited states, the $l=0$ ground state will be the lowest. The $\left(\Gamma_{8}, J=\frac{3}{2}\right)$ heavyhole state will be next. The states associated with the lighthole, $\left(\Gamma_{7}, J=\frac{1}{2}\right)$ and $\left(\Gamma_{6}+\Gamma_{8}, J=\frac{5}{2}\right)$, will be higher in energy. Reference 8 showed from experiment that in InP the $\Gamma_{7}$ state was lower in energy than the $\Gamma_{6}+\Gamma_{8}$ state; the reverse ordering was predicted by theory. ${ }^{9}$ The symmerty of these states was determined using group theory.

Another model was proposed by Rorison et al. ${ }^{10}$ to explain their high-magnetic-field results in InP. In this model 
$D^{0}, X$ is considered to be a free exciton orbiting a neutral donor; one electron was considered to be strongly correlated with the hole and the other with the donor. This model was capable of explaining the relative intensities of the PL transitions in the ground- and excited-state regions of InP.

In this paper we show a detailed magnetic-field splitting of the two states $\left(\Gamma_{6}+\Gamma_{8}, J=\frac{5}{2}\right)$ and $\left(\Gamma_{7}, J=\frac{1}{2}\right)$ associated with the light-hole mass. The splittings are observed in low magnetic fields where there is no overlapping of the states. A nomogram for the interpretation of the magnetic-field components for both states is presented which shows very good agreement with the predicted multiplicity of both states. From the nomographic projection, a light-hole $g_{h 1 / 2}$ value of 0.18 was obtained for the $\Gamma_{7}$ state with a light-hole $g_{h 1 / 2}$ value of 0.26 and a $g_{h 3 / 2}$ value of 0.8 for the $\Gamma_{6}+\Gamma_{8}$ state. The magnetic-field splittings also show that these two states have the same energy ordering as predicted by theory. ${ }^{9} \mathrm{We}$ also show the presence of the rotator states with the terminal excited donor states. We report experimental data for the excited donor states up to $4 D_{+2}$, and compare these with the results of a variational calculation.

\section{EXPERIMENTAL METHOD}

The samples used in this experiment were high-purity GaAs epitaxially grown on semi-insulating GaAs:Cr substrates by means of the $\mathrm{H}_{2}: \mathrm{AsCl}_{3}$ :Ga vapor deposition technique. Hall and electrical conductivity measurements were used to characterize the electrical parameters of the samples. Typical 77-K electron mobilities and carrier concentrations were $1.4 \times 10^{5} \mathrm{~cm}^{2} / \mathrm{V} \mathrm{s}$ and $1.3 \times 10^{14} \mathrm{~cm}^{-3}$, respectively. The PL spectra were excited with a krypton laser. The measurements were performed in the Voigt configuration using a high-resolution 4-m spectrograph. The measurements were made at $2 \mathrm{~K}$ with the sample immersed in liquid He. The emission data were photographically recorded. Magneticfield measurements were made by inserting the tip of the Dewar in the air gap of a conventional dc electromagnet. The maximum field strength of this magnet was $40 \mathrm{kG}$.

\section{RESULTS AND DISCUSSION}

The transitions that are being observed in this experiment result from the collapse of excitons bound to neutral donors. The initial state consists of the bound exciton complex, and the final state consists of the donor electron being either in the ground $D^{0}$ state or an excited state $D^{0 *}$ as shown in Fig. 1. In the presence of an external magnetic field both the initial and the final states will experience splitting. The transitions due to the collapse of an exciton originating in the initial state, where it is associated with the donor ground state and several excited rotational states, and terminating in the donor ground state, are shown in Fig. 2. The states are labeled by their symmetry, and their energy ordering is shown. We speculate that the two highest-energy excited states are associated with the orbital angular momentum $l=2$. The magnetic-field splitting of the $\left(\Gamma_{6}+\Gamma_{8}, J=\frac{5}{2}\right)$ state and the $\left(\Gamma_{7}, J=\frac{1}{2}\right)$ state, both associated with the light-hole mass, was examined in fields varying from 0 to $16 \mathrm{kG}$. The splitting of these two states, whose energy ordering was reversed in InP, is shown in Fig. 3. The dots show the experimental

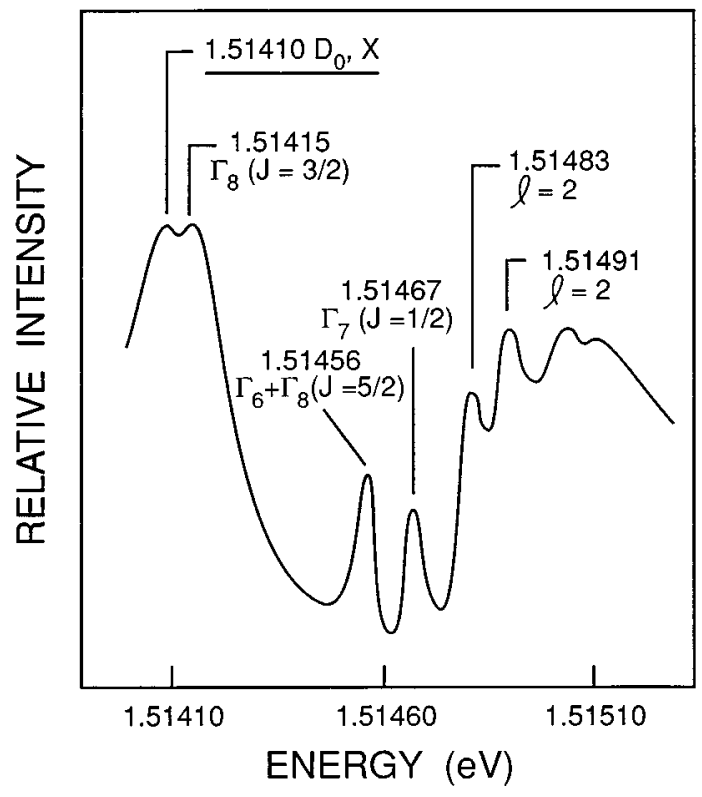

FIG. 1. Ground and excited initial states and ground and excited terminal states of the donor bound exciton in GaAs.

points; the solid lines are added to aid the eye, but have no theoretical significance. It is clear that the $\left(\Gamma_{6}+\Gamma_{8}, J=\frac{5}{2}\right)$ state has a larger number of spin states than the $\left(\Gamma_{7}, J=\frac{1}{2}\right)$ state. The splittings were monitored from the proposed transition scheme shown in Fig. 4.

We have used a geometric construction to analyze the magnetic-field splitting of the above two states. Since the excitons are loosely bound to the shallow donors, it is reasonable to assume that the electron $g$ value in these bound states will be essentially the same as for the free electron. Using an electron $g$ value of $g_{e}=-0.5$, we produced the nomogram for the $\left(\Gamma_{6}+\Gamma_{8}, J=\frac{5}{2}\right)$ state at $16 \mathrm{kG}$ shown in Fig. 5. From this nomogram the hole $g$ values can be determined; $g_{h 3 / 2}$ was determined to be 0.8 , while $g_{h 1 / 2}$ is 0.26 . We define $g_{h 1 / 2}$ from the expression $\Delta E_{1 / 2}=g_{h 1 / 2} \beta H$, where $\Delta E_{1 / 2}$ is the magnetic-field splitting of the $m_{j}= \pm \frac{1}{2}$ hole spin states, and $\beta$ is the Bohr magneton. Similarly, $g_{h 3 / 2}$ is defined using the expression $\Delta E_{3 / 2}=g_{h 3 / 2} \beta H$, where $\Delta E_{3 / 2}$ is the splitting of the $m_{j}= \pm \frac{3}{2}$ states. We also observe a diamag-
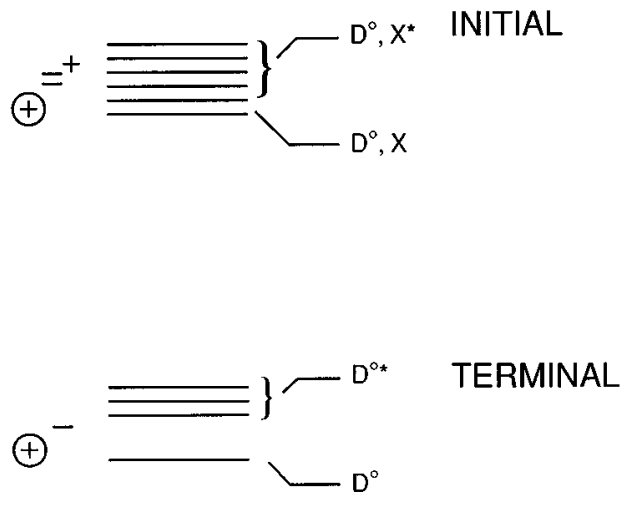

FIG. 2. Photoluminescence showing transitions from initial ground and excited states to the terminal donor ground state in GaAs. 


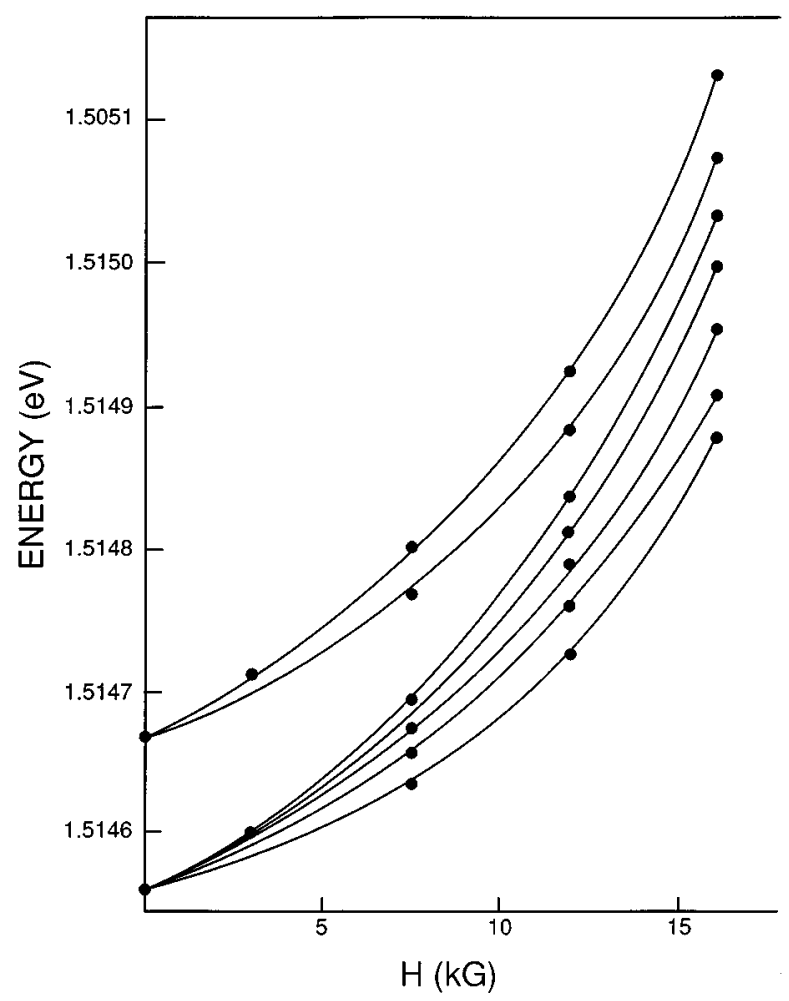

FIG. 3. Magnetic-field splitting of the $\Gamma_{7}$ and $\Gamma_{6}+\Gamma_{8}$ light-hole rotator states.

netic splitting $\Delta$ between the $m_{j}= \pm \frac{1}{2}$ and $m_{j}= \pm \frac{3}{2}$ states. The splittings of the $\left(\Gamma_{6}+\Gamma_{8}, J=\frac{5}{2}\right)$ hole spin states are shown on the upper horizontal line (doubled energy scale). The experimental energies of the spin states are shown on the

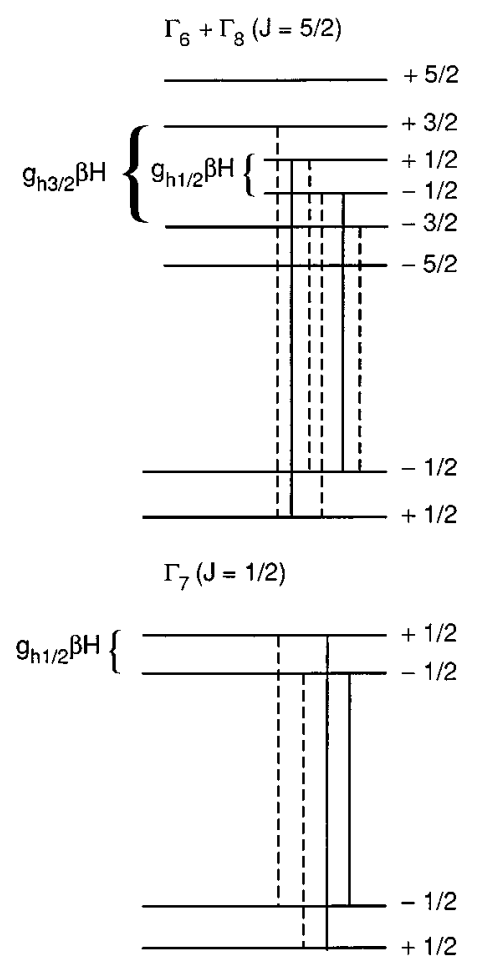

FIG. 4. Energy-level diagram for the principal transitions associated with the $\Gamma_{7}$ and $\Gamma_{6}+\Gamma_{8}$ light-hole rotator states.

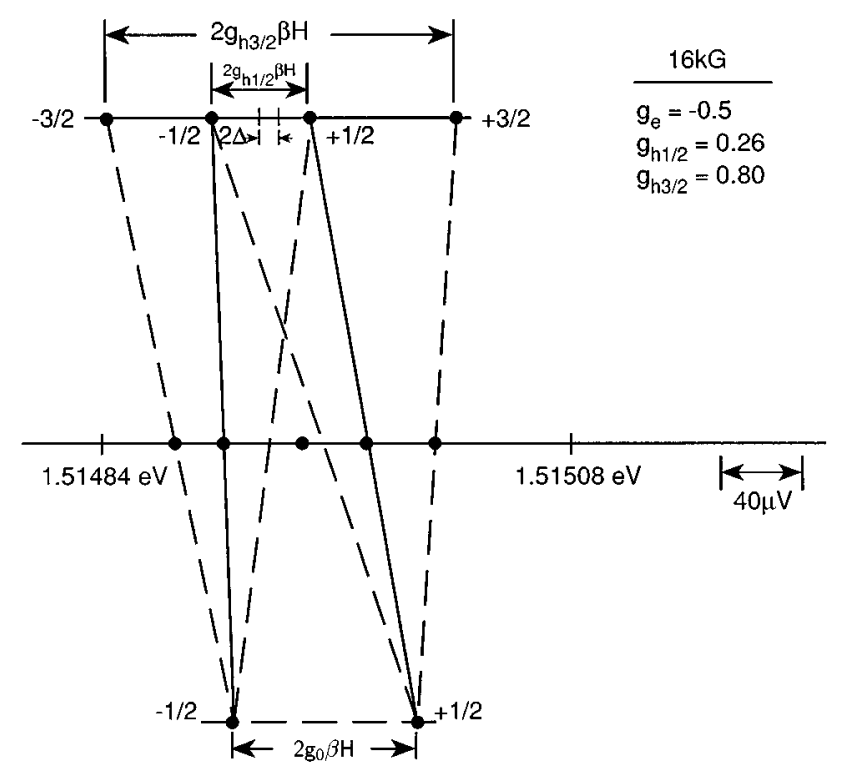

FIG. 5. Nomogram for the Zeeman splitting of the $\left(\Gamma_{6}+\Gamma_{8}, J=\frac{5}{2}\right)$ rotator state. The lines connecting the initial and terminal states intersect the middle line at the experimentally observed energy positions.

center horizontal line (energy increasing to the right). On the lower horizontal line the donor spin states are shown (doubled energy scale). Excellent agreement between all of the intersecting points and the experimental points is observed.

Using the same procedure the nomogram for the $\left(\Gamma_{7}, J=\frac{1}{2}\right)$ state was produced as shown in Fig. 6 . Here the $\left(-\frac{1}{2}-+\frac{1}{2}\right)$ and $\left(+\frac{1}{2}--\frac{1}{2}\right)$ transitions are not observed. The spin conserving transitions would be expected to be the most intense transitions, and it is also noted that the spin-flip transitions are not displaced by more than $10 \mu \mathrm{V}$ from the spin-

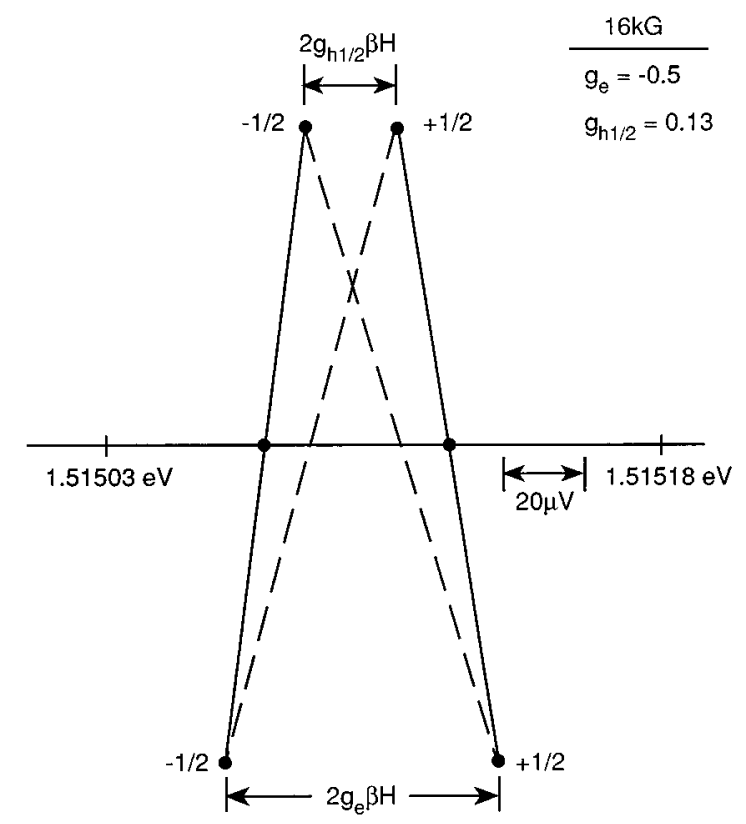

FIG. 6. Nomogram for the Zeeman splitting of the $\left(\Gamma_{7}, J=\frac{1}{2}\right)$ rotator states. The connecting lines have the same function as in Fig. 5. 


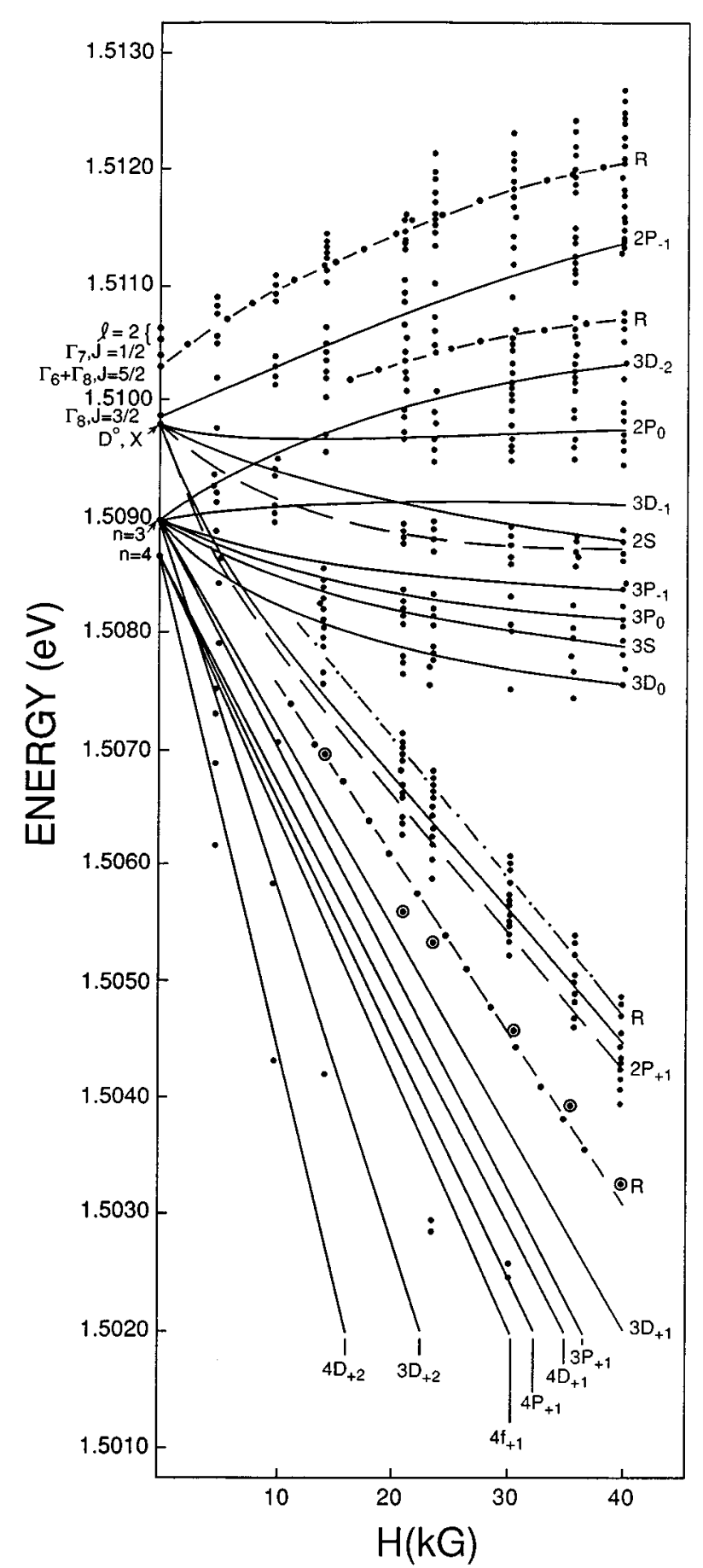

FIG. 7. Transitions associated with the collapse of the exciton in an initial ground or excited state, and terminating in various donor excited states.

conserving transitions. This suggests that there is not a clear resolution of these transitions. From this nomogram the hole $g_{h 1 / 2}$ value was determined to be 0.18 . Based on these measurements, it is clear that the energy ordering shown in Fig. 2 is correct.

The transitions due to the collapse of the exciton in an initial state, in which it is associated with the donor ground state and several excited rotational states, and terminating in various donor excited states, are shown in Fig. 7. In zero magnetic field the donor states are labeled in accordance

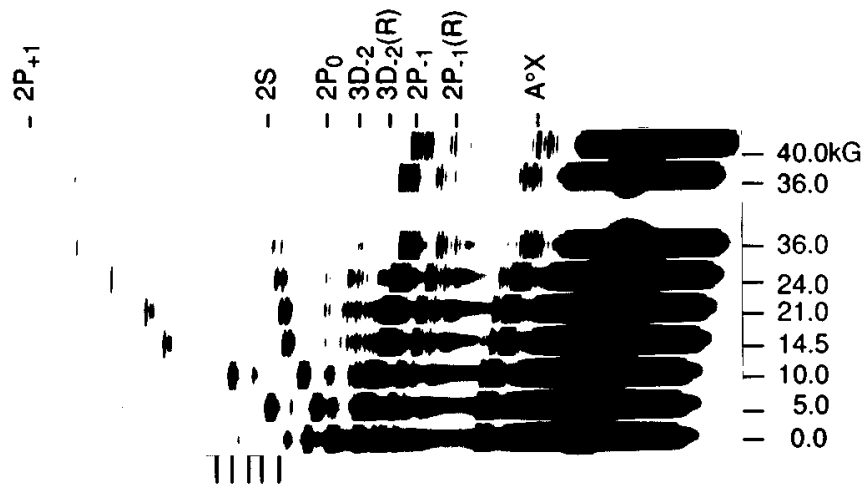

HIGHER EXCITED STATES

FIG. 8. Reproduction of a representative photographic plate recording for a given exposure time.

with the designations given them in Fig. 2, where the final state is the donor ground state. The dots in this figure are the experimental points taken from the photographic plate on which the data were recorded. A reproduction of one of the plates is shown in Fig. 8. The magnetic field is varied from zero to $40 \mathrm{kG}$, and the orbital angular momentum states are labeled as shown.

The labeling of the states with different values of the $z$ component of the total angular momentum such as,- 0 , and + follows the scheme used by Aldrich and Greene. ${ }^{11}$ In Fig. 7 the solid lines with different values of the $z$ component of the total angular momentum are obtained as differences between the calculated values of Aldrich and Greene ${ }^{11}$ and their value of the $2 P_{-1}$ level. The experimental $2 P_{+1}$ state deviates slightly from the theory, and is shown as the dashed line. The experimental $2 S$ state (again shown as a dashed line) shows a larger value of the curvature at low magnetic fields than that obtained from theory. The energy differences are, however, rather small. Different exposure times are required to record the spectra, so that some of the more intense states must be overexposed in order to record the less intense states, and overlapping makes it impossible to resolve all of the spin states. Rotator states (dot-dashed curves) associated with the $2 P_{-1}, 3 D_{-2}$, and $2 P_{+1}$ can be followed in Fig. 7 as the magnetic field increases. The exciton collapses from the various excited rotational states in the initial state, and places the donor electron in the above excited states in the final state. It is quite likely that there are rotator states associated with all of the excited donor states, but which are not resolved because of overlap. It is noted that the diamagnetic shift of the rotator states associated with the $2 P_{-1}$ state is greater than that of the $2 P_{-1}$ state associated with the ground state of $D^{0}, X$. This would be expected since the rotator states have smaller binding energies than the $D^{0}, X$ state. Some rather broad transitions, shown as open circles, are observed between the $2 P_{+1}$ and $3 D_{+1}$ states. There are no excited-state transitions predicted theoretically in this energy region. We speculate that these transitions result from the rotator state transitions in which the rotators are associated with the $n=3$ excited state of the donor bound exciton. These transitions are also shown as dot-dashed curves. The MPL spectra at low magnetic fields are particularly successful in depicting the 
evolution of ground and excited donor bound exciton states as well as the excited rotator states.

\section{SUMMARY}

We have observed magnetic-field splitting of the two rotator states designated as $\left(\Gamma_{6}+\Gamma_{8}, J=\frac{5}{2}\right)$ and $\left(\Gamma_{7}, J=\frac{1}{2}\right)$ associated with donor bound excitons in high-purity epitaxial GaAs in magnetophotoluminescence spectra. These two states are associated with the light-hole mass and are split by the spin-orbit interaction. The ordering in energy of these two states agrees with that predicted by theory. We also observed the rotator states in which, after the radiative collapse of the exciton, the donor is left in the excited states. A good agreement between the energies of the excited states of the donor, as determined experimentally with those calculated using a variational approach, is found.

\section{ACKNOWLEDGMENTS}

This work was partially supported by the Air Force Office of Scientific Research (AFOSR). The work of D.C.R., D.C.L., and B.J. was performed at Wright Laboratory, Solid State Electronics Directorate (WL/EL), Wright Patterson Air Force Base under U.S.A.F. Contract No. F33615-91-C-1765.
${ }^{1}$ R. J. Almassy, D. C. Reynolds, C. W. Litton, K. K. Bajaj, and G. L. McCoy, Solid State Commun. 38, 1052 (1981).

${ }^{2}$ D. C. Reynolds, K. K. Bajaj, C. W. Litton, and E. B. Smith, Phys. Rev. B 28, 3300 (1983).

${ }^{3}$ S. S. Bose, B. Lee, M. H. Kim, and G. E. Stillman, Appl. Phys. Lett. 51, 937 (1987).

${ }^{4}$ S. P. Watkins, G. Haacke, and H. Burrhard, Appl. Phys. Lett. 52, 401 (1988).

${ }^{5}$ T. D. Harris, M. S. Skolnick, J. M. Parsey, Jr., and R. Bhat, Appl. Phys. Lett. 52, 389 (1988).
${ }^{6}$ P. J. Dean, D. C. Herbert, and A. M. Lahee, J. Phys. C 13, 5071 (1980).

${ }^{7}$ C. Benoit à la Guillaume and P. Lavallard, Phys. Status Solidi B 70, K143 (1975).

${ }^{8}$ W. Rühle and W. Klingenstein, Phys. Rev. B 18, 7011 (1977).

${ }^{9}$ J. C. Herbert, J. Phys. C 10, 3327 (1977).

${ }^{10}$ J. Rorison, D. C. Herbert, P. J. Dean, and M. S. Skolnick, J. Phys. C 17, 6453 (1984).

${ }^{11}$ C. Aldrich and R. L. Greene, Phys. Status Solidi B 93, 343 (1974). 